\title{
On Geometric Phases for Soliton Equations
}

\author{
M.S. Alber ${ }^{1}$ and J.E. Marsden ${ }^{2}$ \\ ${ }^{1}$ Department of Mathematics, University of Notre Dame, Notre Dame, IN 46556, USA \\ 2 Department of Mathematics, University of California, Berkeley, CA 94720, USA
}

Received April 1, 1991; in revised form March 27, 1992

\begin{abstract}
This paper develops a new complex Hamiltonian structure for $n$-soliton solutions for a class of integrable equations such as the nonlinear Schrödinger, sine-Gordon and Korteweg-de Vries hierarchies of equations that yields, amongst other things, geometric phases in the sense of Hannay and Berry. For example, one of the possible soliton geometric phases is manifested by the well known phase shift that occurs for interacting solitons. The main new tools are complex angle representations that linearize the corresponding Hamiltonian flows on associated noncompact Jacobi varieties. This new structure is obtained by taking appropriate limits of the differential equations describing the class of quasi-periodic solutions. A method of asymptotic reduction of the angle representations is introduced for investigating soliton geometric phases that are related to the presence of monodromy at singularities in the space of parameters. In particular, the phase shift of interacting solitons can be expressed as an integral over a cycle on an associated Riemann surface. In this setting, soliton geometric asymptotics are constructed for studying geometric phases in the quantum case. The general approach is worked out in detail for the three specific hierarchies of equations mentioned. Some links with $\tau$-functions, the braid group and geometric quantization are pointed out as well.
\end{abstract}

\section{Introduction}

We begin by summarizing a few recent developments in the theory of geometric phases that are relevant to the present paper. In [12] Berry considered a geometric phase factor $\exp (i \gamma)$ (in addition to the dynamical phase) for systems that are slowly transported along a closed curve in a space of parameters. In [41] a class of connections was constructed to obtain expressions for the Hannay-Berry phases [14] (geometric angle shifts in the classical case) for some integrable problems in terms of the non-trivial holonomy of these connections. In [41], Montgomery gave an example of a phase that is linked with singularities in the case of a flat connection. Symmetry and reduction were used to obtain a generalization of 
geometric phases to the non-integrable case in the form of the holonomy of the Cartan-Hannay-Berry connection [37]. It was shown in [6] that geometric phases for problems with singularities on hyperelliptic Jacobian fibrations can be connected with the symplectic representation of the braid group $[3,11]$. The fact that action-angle variables could be used for quantization of classical systems $[33,5]$ leads to the introduction of semiclassical geometric phases $[13,6]$. For algebraic completely integrable systems [4], such variables can be obtained by generalizing the definition of actions introduced for integrable systems on tori in the form of periods of holomorphic differentials along the elements of a homology basis $[10,31]$. For discrete problems, action-angle maps were explicitly constructed in particular, for the Toda lattice [26] and in the case of Calogero-Moser systems and their corresponding relativistic generalizations [45]. In [20] and [35] it is shown that possible obstructions to the existence of global systems of action-angle variables on symplectic vector bundles are a nontrivial first Chern class and the presence of monodromy at singularities.

All of the papers mentioned above deal with problems that have compact invariant varieties. The goal of the present paper is to use complex varieties to gain insight into the theory of geometric phases for nonlinear systems that have noncompact varieties. Our general method will be demonstrated for specific soliton equations.

In Sect. 5.2 we introduce the method of "asymptotic reduction" of the angle representations on Jacobi varieties to obtain Hamiltonian integrable flows in the noncompact case. In particular, it yields finite-dimensional Hamiltonians and an angle representation for the $n$-soliton solutions of nonlinear systems; they are defined on topologically nontrivial phase spaces. Then we obtain soliton geometric phases in the classical case and relate some of them to the soliton phase shift.

In the context of phases one usually puts a given system in action-angle form and considers a shift in the angle variables $\theta=\left(\theta_{1}, \ldots, \theta_{n}\right)$ after transporting the system along a closed curve in the space of parameters. This change consists of two parts,

$$
\Delta \theta=\Delta_{D} \theta+\Delta_{G} \theta
$$

called the dynamic and geometric phases. The dynamic phase is due to the dynamical evolution of the system and it is proportional to the period of time $(T)$ during which system is transported along the closed curve. To eliminate the dynamical phase and to retain only the geometric part, the method of averaging is usually used. In the soliton case, the period is infinite and so we use the method of "asymptotic reduction" and the complex phase function instead of the averaging approach.

There are three different approaches to soliton geometric phases described in this paper.

1. The first approach uses the fact that our angle-representation is related to the Abel-Jacobi map and that asymptotic reduction leads to the complex splitting of the spectrum of the soliton problem. Time is considered to be a parameter of the system.

2. The second method uses averaged differentials of the angle variables in the space of parameters and analytic continuation of the soliton phase function into the complex domain. Phases are then obtained as a monodromy at singularities of the 
phase function considered as a function of several complex variables. As a result, it describes the geometric phase in terms of certain invariants of the Riemann surface (corresponding to a conserved level set of the concrete integrable problem). This phase is independent of the initial conditions.

3. The third way is to use a relation between the $\theta$-function and $\tau$-function, which gives a link between the phase shifts of solitons, soliton geometric phases and geometric phases for quasi-periodic solutions (see [6]). At the same time, this enables one to introduce and investigate phases in the general case of soliton solutions of Hirota equations [2] connected with the representation theory of Kac-Moody algebras.

These methods enable one to introduce and investigate phases in the complex case and yield a new class of geometric phase phenomena that can be also understood in terms of the deformation of an associated $n$-dimensional noncompact Jacobi variety. The phase space considered previously [14] in the context of Hannay-Berry phases was usually foliated by a family of Jacobi tori.

Our general approach is demonstrated for the $n$-soliton solutions of the Nonlinear Schrödinger (NLS), sine-Gordon (SG) and Korteweg-de Vries (KdV) hierarchies of equations. In particular, in case of the focusing NLS equations, asymptotic reduction of the angle representation yields a term that describes the remaining interaction after the asymptotic splitting of the $n$-soliton solution. It makes the case of the (focusing) (f) NLS equation different from other soliton equations and might have applications in nonlinear optics.

Complex geometry related to the $\theta$-functions was previously studied in the $\mathrm{KdV}$ case by McKean and Ercolani [39, 21]. In [28], the class of soliton equations associated with the AKNS eigenvalue problem was shown to be generated by a family of commuting infinite-dimensional Hamiltonians on a subalgebra of the loop algebra of $\operatorname{sl}(2)$. The modulational Poisson structure [22] for the sineGordon system was derived in terms of conformal ingredients such as differentials on Riemann surfaces and $\theta$-functions and a possible link with the Hamiltonian theory was investigated.

The finite dimensional complex Hamiltonians and phases obtained in this paper can be also used for investigating modulation equations.

Lastly, our soliton Hamiltonian structures lead to the introduction of presoliton geometric asymptotics and using the results described above, we obtain a link with geometric phases in the quantum case.

\section{Preliminaries}

This section reviews some results from the theory of soliton equations. Recall that $n$-soliton solutions or reflectionless potentials were first obtained as a special class of solutions of the Korteweg-de Vries $(\mathrm{KdV})$ equation

$$
U_{t}+6 U U_{x}+U_{x x x}=0
$$

and that these solutions allow the collision of solitons. Collisions are special because after collision the solitons are, as is well known, of the same form as before. The only parameter in the multi-soliton solution which is changed is the so-called phase of the $k^{\text {th }}$ soliton, namely the change of the coordinate of the center of the $k^{\text {th }}$ 
soliton after the collision that is additional to the constant velocity motion. The formula for the phase shift of the soliton was obtained using asymptotic methods $[1,2,17]$.

In $[2,44]$ soliton solutions were investigated for the nonlinear Schrödinger (NLS) equations

$$
i \dot{Q}+\frac{1}{2} Q^{\prime \prime}-\bar{Q} Q^{2}=0, \quad \text { the defocusing case: (d) NLS }
$$

and

$$
i \dot{Q}+\frac{1}{2} Q^{\prime \prime}+\bar{Q} Q^{2}=0, \quad \text { the focusing case: (f) NLS }
$$

and for the sine-Gordon (SGE) equation [29]

$$
U_{x x}-U_{t t}=\sin U
$$

which have important applications in nonlinear optics (see [30, 34]).

We start by recalling some of the different methods available for constructing many-soliton solutions. First of all, they can be obtained by the inverse scattering transform in the form of reflectionless potentials [2]. Second, they can be constructed in terms of $\tau$-functions from quasi-periodic (algebraic-geometric) solutions $[39,38,40]$ of the integrable problem as a result of a special limiting process applied to a quasi-periodic potential represented in terms of $\theta$-functions. This limiting process and $\tau$-functions on the Jacobi cylinder were investigated in detail by McKean [39] for the KdV equation. The geometry of $\theta$-functions in the periodic case for $\mathrm{KdV}$ was studied in [21]. (For details about $\theta$-functions see Mumford [43] and for the limiting process in the NLS case see [44]).

On the other hand a connection was established (see [47]) between modulation theory [27] and the small dispersion limit [36]. Moreover, the $\theta$-function of the periodic theory was obtained [48] in the form of a singular limit of the Dyson determinant.

\section{Complex Quasi-Periodic Hamiltonian Systems}

It is known [38, 40,9] that there are finite dimensional invariant tori in the phase space of completely integrable nonlinear problems. Solutions lying on these tori, called quasi-periodic solutions, can be described using a pair of commuting Hamiltonian systems written in configuration variables $\left(\lambda_{1}, \ldots, \lambda_{n}\right)$ and momentum variables $\left(P_{1}, \ldots, P_{n}\right)$ with Hamiltonian of the form

$$
H^{s}=\sum_{j=1}^{n} \frac{\left(P_{j}^{2}+C\left(\lambda_{j}\right)\right)}{\prod_{r \neq j}\left(\lambda_{j}-\lambda_{r}\right)} F_{j}^{s}
$$

and

$$
H^{d}=\sum_{j=1}^{n} \frac{\left(P_{j}^{2}+C\left(\lambda_{j}\right)\right)}{\prod_{r \neq j}\left(\lambda_{j}-\lambda_{r}\right)} F_{j}^{d},
$$


where the functions $C(\lambda)$ and $F_{j}^{s}$ and $F_{j}^{d}$ are specified below. Both these Hamiltonians have a common set of first integrals, i.e., associated conservation laws,

$$
P_{j}^{2}=-C\left(\lambda_{j}\right)=-\prod_{k=1}^{N}\left(\lambda_{j}-m_{k}\right), \quad j=1, \ldots, n .
$$

Note that these Hamiltonians have terms quadratic in their momenta and can be written in the form

$$
H=\frac{1}{2} \sum_{j=1}^{n} g^{j j} P_{j}^{2}+V\left(\lambda_{1}, \ldots, \lambda_{n}\right) .
$$

We regard Hamiltonian systems as defined on the complex phase space $C^{2 n}$ with the complex symplectic structure

$$
\Omega=\sum_{j=1}^{n} d P_{j} \wedge d \lambda_{j}
$$

and with the Hamiltonian and the integrals depending on the parameters $m_{1}, \ldots, m_{N}$. Here

$$
\begin{gathered}
N=2 n+2, \quad F_{j}^{s}=1, \quad F_{j}^{d}=\left(-\sum_{l \neq j} \lambda_{l}+\frac{1}{2} \sum_{k=1}^{2 n+2} m_{k}\right) \text { for the NLS case } \\
F_{j}^{s}=1+G_{n-1}, \quad F_{j}^{d}=1-G_{n-1}, C(\lambda)=\frac{1}{\lambda} \prod_{k=1}^{2 n}\left(\lambda-m_{k}\right) \text { for the sine-Gordon case, } \\
N=2 n+1, \quad F_{j}^{s}=1, \quad F_{j}^{d}=2\left(-\sum_{l \neq j} \lambda_{l}+\frac{1}{2} \sum_{k=1}^{2 n+1} m_{k}\right) \text { for the KdV case }
\end{gathered}
$$

The sine-Gordon case will be discussed in detail in Sect. 6.4.

We can regard each expression (3.5) as being defined on the Riemannian surface

$$
\mathfrak{R}: P^{2}=-C(\lambda) \text {. }
$$

Considered together, they determine an $n$-dimensional complex Lagrangian manifold

$$
\Gamma:(\Re \times \ldots \times \Re) / \sigma_{n},
$$

where $\sigma_{n}$ is the permutation group on $n$ letters and with generating function

$$
S=\sum_{j=1}^{n} \int_{\lambda_{j}^{o}}^{\lambda_{j}} P_{j} d \lambda_{j}=\sum_{j=1}^{n} \int_{\lambda_{j}^{o}}^{\lambda_{j}} \sqrt{-C\left(\lambda_{j}\right)} d \lambda_{j} .
$$

The $\lambda_{j}$ are the so-called root-variables, which in the real case vary along cycles $l_{j}$ over basic cuts $\left[m_{2 j}, m_{2 j-1}\right]$,

$$
m_{2 n+1}<m_{2 n}<\ldots<m_{1}, \quad m_{k}=\text { const } .
$$

on the Riemann surfaces (3.7). Here we consider the general case of complex polynomials and regard $\lambda_{j}$ as being defined on the Riemann surfaces. In terms of these variables, one can reconstruct the potentials $U$ and $Q$ described above. For 
example, the quasi-periodic potential (2.1) in the $\mathrm{KdV}$ case has the following form:

$$
U=-2 \sum_{j=1}^{n} \lambda_{j}(x, t)+\sum_{k=1}^{2 n+1} m_{k} .
$$

Here, $\lambda_{j}(x, t)$ is defined as follows. The functions $H^{s}$ and $H^{d}$ generate two commuting Hamiltonian flows describing the spatial and temporal evolutions. In other words, one gets a solution $\lambda_{j}$ starting at a particular value $\left(x_{0}, t_{0}\right)$ for all values of $(x, t)$ by flowing successively by the flow of the Hamiltonian vector field of $H^{s}$ and then $H^{d}$ or vice versa.

In what follows, we consider certain limiting systems obtained from quasiperiodic systems of equations and introduce a new soliton phase space together with a system of action-angle variables and describe the corresponding finitedimensional soliton Hamiltonians. The results obtained give an example of a system with action-angle variables, but without invariant tori and yield the soliton symplectic structure. This provides a setting in which we can introduce soliton geometric phases by investigating the behavior of such systems of coordinates in the neighbourhoods of singular points in the space of parameters. This problem is defined on the generalized Jacobi variety of the symmetric product of $n$ logarithmic Riemannian surfaces in place of the Liouville tori.

\section{Soliton Hamiltonian Systems}

Here we introduce the soliton phase space and, on it, define new finite-dimensional soliton Hamiltonians. In what follows we describe the NLS(d) and KdV cases in detail and then state the corresponding results for the NLS(f) and sine-Gordon equations.

To obtain the soliton problem we shrink the so-called allowed zones [39]

$$
\left.\begin{array}{l}
{\left[m_{2 k+1}, m_{2 k}\right] \rightarrow a_{k}, a_{k}=\left(i\left(\alpha_{k}\right)\right)^{2}, k=1, \ldots, n} \\
\mathrm{KdV}: m_{1} \rightarrow a_{0}=0, a_{n+1}=0, a_{n}<\ldots<a_{1}<a_{0}=0, \\
(d) \mathrm{NLS}: m_{1} \rightarrow b_{1}, m_{2 n+2} \rightarrow b_{2},\left(a_{n+1}=b_{2}\right)<a_{n}<\ldots<a_{1}<\left(a_{0}=b_{1}\right)<0 .
\end{array}\right\}
$$

As a result of the limiting process (4.1), the expression (3.11) yields the following formula for multi-soliton solutions:

$$
U=-2 \sum_{j=1}^{n} \lambda_{j}(x, t)+2 \sum_{k=1}^{n} a_{k}+a_{0}+a_{n+1} .
$$

From the Hamiltonian systems with Hamiltonians (3.3) and (3.4) we obtain equations for the soliton root-variables $\left\{\lambda_{j}\right\}$ as

$$
\begin{aligned}
& \frac{\partial \lambda_{j}}{\partial x}=2 \frac{M\left(\lambda_{j}\right) \prod_{k=1}^{n}\left(\lambda_{j}-a_{k}\right)}{\prod_{i \neq j}\left(\lambda_{j}-\lambda_{i}\right)}, j=1, \ldots, n, \\
& \frac{\partial \lambda_{j}}{\partial t}=2\left(-\sum_{l \neq j}^{n} \lambda_{l}+\sum_{k=0}^{n+1} a_{k}\right) \frac{M\left(\lambda_{j}\right) \prod_{k=1}^{n}\left(\lambda_{j}-a_{k}\right)}{\prod_{i \neq j}\left(\lambda_{j}-\lambda_{i}\right)} .
\end{aligned}
$$


Here

$$
\left.\begin{array}{l}
\operatorname{KdV}: M\left(\lambda_{j}\right)=\sqrt{-\lambda_{j}} \\
(d) \operatorname{NLS}: M\left(\lambda_{j}\right)=\sqrt{-\left(\lambda_{j}-b_{1}\right)\left(\lambda_{j}-b_{2}\right)}
\end{array}\right\}
$$

Theorem 4.1. The systems (4.3) and (4.4) are soliton Hamiltonian systems with Hamiltonians

$$
H_{s}^{s}=\frac{\sum_{j=1}^{n}\left(e^{2 M\left(\lambda_{j}\right) P_{j}}-\left(\bar{C}\left(\lambda_{j}\right)\right)^{1 / 2} / M\left(\lambda_{j}\right)\right)}{\prod_{r \neq j}\left(\lambda_{j}-\lambda_{r}\right)}
$$

and

$$
H_{s}^{d}=\frac{\sum_{j=1}^{n}\left(-\sum_{l \neq j} \lambda_{l}+\sum_{k=0}^{n+1} a_{k}\right)\left(e^{2 M\left(\lambda_{J}\right) P_{J}}-\left(\bar{C}\left(\lambda_{j}\right)\right)^{1 / 2} / M\left(\lambda_{j}\right)\right)}{\prod_{r \neq j}\left(\lambda_{j}-\lambda_{r}\right)}
$$

and with first integrals

$$
P_{j}=\frac{\sum_{k=1}^{n} \log \left(\lambda_{j}-a_{k}\right)}{2 M\left(\lambda_{j}\right)} j=1, \ldots, n .
$$

Here $\bar{C}(E)=\left(M(E) \prod_{k=1}^{n}\left(E-a_{k}\right)\right)^{2}$ is a polynomial with constant coefficients obtained as a result of the shrinking the basic polynomial $C(E)$ of the Riemann surface. The expression (4.6) can be considered as a constraint for (4.7).

Proof. Substituting the expressions (4.8) into the first part of the Hamiltonian system

$$
\begin{aligned}
& \lambda_{j}^{\prime}=\frac{\partial \lambda_{j}}{\partial x}=\frac{\partial H_{S}^{S}}{\partial P_{j}}\left(\text { or } \quad \dot{\lambda}_{j}=\frac{\partial \lambda_{j}}{\partial t}=\frac{\partial H_{S}^{d}}{\partial P_{j}}\right), \\
& P_{j}^{\prime}=\frac{\partial P_{j}}{\partial x}=-\frac{\partial H_{S}^{S}}{\partial \lambda_{j}} \quad\left(\text { or } \quad \dot{P}_{j}=\frac{\partial P_{j}}{\partial t}=-\frac{\partial H_{S}^{d}}{\partial \lambda_{j}}\right)
\end{aligned}
$$

we get the systems (4.3) and (4.4).

The first integrals (4.8) define a Lagrangian submanifold of the phase space $C^{2 n}$ in the form of the symmetric product

$$
\Gamma:(\mathfrak{R} \times \ldots \times \mathfrak{R}) / \sigma_{n}
$$

of $n$ copies of the Riemann surface

$$
\mathfrak{R}: P=\frac{\sum_{k=1}^{n} \log \left(\lambda-a_{k}\right)}{2 M(\lambda)} .
$$

Notice that if we took a formal limit of the Hamiltonian structure, we would produce a phase space with singularities (i.e., a pinched torus). The Hamiltonian structure obtained here is presumably a regularisation of the pinched torus in an appropriate sense, but that aspect is not discussed here.

Remark 4.2. A special class of the solutions of the $\mathrm{KdV}$ equation corresponding to the case when some of the parameters $a_{r}$ are positive can be obtained using the same approach. These solutions by analogy with the (d)NLS equation are called "dark-hole" solitons for the $\mathrm{KdV}$ equation. 


\section{Angle Representations on Noncompact Jacobi Varieties and Asymptotic Reduction}

Now we construct systems of action-angle variables on the soliton phase space and obtain the corresponding soliton geometric phases.

5.1. Angle-Representations and Noncompact Jacobi Varieties. There are no invariant tori in the soliton case and therefore we cannot use the usual actions $[10,31]$. Nevertheless, it is possible to introduce variables that linearize the soliton Hamiltonian flows on the phase spaces described in the previous section.

To do so, we consider the action function

$$
S=\sum_{j=1}^{n} \int_{\lambda_{j}^{o}}^{\lambda_{j}} P_{j} d \lambda_{j}=\sum_{j=1}^{n} \frac{1}{2} \int_{\lambda_{j}^{o}}^{\lambda_{j}}\left(\frac{\sum_{k=1}^{n} \log \left(\lambda_{j}-a_{k}\right)}{M\left(\lambda_{j}\right)}\right) d \lambda_{j},
$$

and introduce conjugate variables $\overline{I_{k}}$ and $\overline{\theta_{k}}$ as follows:

$$
\bar{I}_{k}=a_{k}, \quad \bar{\theta}_{k}=-\frac{\partial S}{\partial \bar{I}_{k}}=\frac{\partial S}{\partial a_{k}}=\sum_{j=1}^{n} \frac{1}{2} \int_{\lambda_{j}^{o}}^{\lambda_{j}} \frac{d \lambda_{j}}{M\left(\lambda_{j}\right)\left(\lambda_{j}-a_{k}\right)} .
$$

Definition 5.1. We call a complete set of variables $\bar{\theta}_{j}, j=1, \ldots, n$, an anglerepresentation of the multi-soliton solution on the associated $n$-dimensional complex Lagrangian submanifolds. It describes a map of Abel-Jacobi type determining a noncompact Jacobi variety.

Theorem 5.2. In terms of the variables $\left(\bar{I}_{k}, \bar{\theta}_{k}\right)$, the soliton Hamiltonian flows are linearized on the noncompact Jacobi variety.

Proof. In what follows we show that $\overline{\theta_{k}}$ are linear in $x$ and $t$. Differentiating (5.2) with respect to $x$ and $t$ and using (4.3), (4.4) one obtains the following system:

$$
\begin{gathered}
\bar{\theta}_{k}^{\prime}=\sum_{j=1}^{n} \frac{\lambda_{j}^{\prime}}{2 M\left(\lambda_{j}\right)\left(\lambda_{j}-a_{k}\right)}=\sum_{j=1}^{n} \frac{\prod_{l=1, l \neq k}^{n}\left(\lambda_{j}-a_{l}\right)}{\prod_{i \neq j}\left(\lambda_{j}-\lambda_{i}\right)}=1, \quad k=1, \ldots, n, \\
\dot{\dot{\theta}_{k}}=\sum_{j=1}^{n} \frac{\dot{\lambda}_{j}}{2 M\left(\lambda_{j}\right)\left(\lambda_{j}-a_{k}\right)}=\sum_{j=1}^{n}\left(-\sum_{l \neq j}^{n} \lambda_{l}+\sum_{r=0}^{n+1} a_{r}\right) \frac{\prod_{l=1, l \neq k}^{n}\left(\lambda_{j}-a_{l}\right)}{\prod_{i \neq j}\left(\lambda_{j}-\lambda_{i}\right)}=a_{k} .
\end{gathered}
$$

The right-hand sides of (5.3) and (5.4) are identically equal to 1 (or $a_{k}$ respectively) for all $k=1, \ldots, n$. After integrating (5.3) and (5.4) the following system results

$$
\overline{\theta_{k}}=x+v_{k} t+\varphi_{k}, \quad \bar{I}_{k}=a_{k}, \quad v_{k}=a_{k} \quad k=1, \ldots, n,
$$

which proves that $\left(\bar{\theta}_{k}, \bar{I}_{k}\right)$ are really action-angle variables of the problem in the sense that the soliton flow linearizes in them.

The linear dependence of $\bar{\theta}_{k}$ on $x$ and $t$ demonstrates the fact that the $x$ and $t$ flows commute with each other.

Corollary 5.3. A complex soliton symplectic structure is given by

$$
\Omega=\sum_{j=1}^{n} d \bar{\theta}_{j} \wedge d \bar{I}_{j}
$$


where $\overline{I_{j}} \Leftarrow a_{j}$ are first integrals of the problem.

This topic will be pursued in a forthcoming paper.

5.2. Asymptotic Reduction. The existence of singularities in the system (5.2) results in the multivaluedness of the system of action-angle variables and represents one of the possible obstructions to the construction of a global system of such variables.

In what follows, we use the angle-representations for different problems to deduce the associated phase functions $\varphi$ on the noncompact Jacobi varieties and we shall introduce and investigate geometric phases in the complex case. These functions also have important applications in the theory of evolution equations (specifically the Whitham equations).

Now we show that the angle-representation allows one to determine the limiting behavior of the $n$-soliton solution and to describe a system of soliton geometric phases. We call the procedure described below the "soliton analysis."

Note that in the general case, basic points $\lambda_{j}^{o}$ of the angle map (5.2) are different from the initial points $\lambda_{j}(0,0)$.

Theorem 5.4. As $(t \rightarrow \infty)$ or $(t \rightarrow-\infty)$ the system of angle variables of the $n$-soliton solution (5.2) splits into $n$ 1-soliton angle variables. We shall call this process asymptotic reduction.

Proof. We consider the expression for the $n$-soliton angle-variables

$$
\overline{\theta_{r}}=\sum_{j=1}^{n} \frac{1}{2} \int_{\lambda_{j}^{o}}^{\lambda_{j}} \frac{d \lambda_{j}}{\left(M\left(\lambda_{j}\right)\left(\lambda_{j}-a_{r}\right)\right)}=x+v_{r} t, \quad r=1, \ldots, n
$$

for the particular choice of initial values of the root-variables

$$
\lambda_{j}(0,0)=\lambda_{j}^{o}=\frac{a_{j-1}+a_{j}}{2} .
$$

We pause in the proof for a remark:

Remark 5.5. Note that term $v_{r} t$ will generate a dynamical soliton phase

$$
\Delta_{D} \overline{\theta_{r}}=v_{r} T .
$$

Here $T$ is in the context of Hannay-Berry phases a period of time during which system is transported along a closed curve in the space of parameters. $\Delta_{D} \bar{\theta}_{r}$ is a shift of the angle variables due to dynamics. Averaging of the angle variables is usually used to eliminate this term and to calculate additional (geometric) phase. Our case is special since we are dealing with the infinite period $T$. Instead of averaging we will use the method of asymptotic reduction.

In what follows we will investigate (5.7) as $(t \rightarrow \infty)$ (or $(t \rightarrow-\infty)$ ). Now we define a direction in the $(x, t)$ plane by fixing

$$
x+v_{k} t=d_{k}=\text { const }
$$


for some $r=k$. Then we transform the $k^{\text {th }}$ equation from (5.7) adding and subtracting integrals along different intervals on the real axis to obtain a complete integral from 0 to $\lambda_{k}$

$$
\left.\begin{array}{r}
\frac{1}{2} \int_{0}^{\lambda_{k}} \frac{d \lambda}{\left(M(\lambda)\left(\lambda-a_{k}\right)\right)}=d_{k}+\frac{1}{2} \sum_{j<k} \int_{\lambda_{j}}^{\lambda_{j+1}^{o}} \frac{d \lambda_{j}}{\left(M\left(\lambda_{j}\right)\left(\lambda_{j}-a_{k}\right)\right)} \\
+\frac{1}{2} \int_{0}^{\lambda_{1}^{o}} \frac{d \lambda_{1}}{\left(M\left(\lambda_{1}\right)\left(\lambda_{1}-a_{k}\right)\right)}-\frac{1}{2} \sum_{j>k} \int_{\lambda_{j}^{o}}^{\lambda_{j}} \frac{d \lambda_{j}}{\left(M\left(\lambda_{j}\right)\left(\lambda_{j}-a_{k}\right)\right)}+\varphi_{k}^{o}
\end{array}\right\} .
$$

Here $\varphi_{k}^{o}$ is a term chosen to be consistent with the initial data (5.8). Notice that in the real case (when all $\lambda_{j}$ and $P_{j}$ are real), every $\lambda_{j}$ is varying once along the cycle $l_{j}$ over the basic cut $\left[a_{j}, a_{j-1}\right]$ on the Riemann surface (4.8) with the $P_{j}$ 's treated as constants.

The rest of the (5.7) can be described for a fixed $d_{k}$ as follows:

$$
\sum_{j=1}^{n} \frac{1}{2} \int_{\lambda_{j}^{o}}^{\lambda_{j}} \frac{d \lambda_{j}}{\left(M\left(\lambda_{j}\right)\left(\lambda_{j}-a_{r}\right)\right)}=x+v_{r} t=d_{k}+\left(v_{r}-v_{k}\right) t, \quad r \neq k, \quad r=1, \ldots, n .
$$

Here $\left(v_{r}-v_{k}\right)>0, r<k$, and $\left(v_{r}-v_{k}\right)<0, r>k$.

System (5.11) yields the following limits

$$
\left.\begin{array}{ll}
\text { 1. } t \rightarrow \infty: \lambda_{r} \rightarrow a_{r-1} \text { for } r>k & \text { and } \lambda_{r} \rightarrow a_{r} \text { for } r<k \\
\text { 2. } t \rightarrow-\infty: \lambda_{r} \rightarrow a_{r} \text { for } r>k & \text { and } \lambda_{r} \rightarrow a_{r-1} \text { for } r<k .
\end{array}\right\}
$$

This transforms (5.10) into the expression for the 1-soliton angle variable

$$
\bar{\Theta}_{k}=\frac{1}{2} \int_{0}^{\lambda_{k}} \frac{d \lambda}{\left(M(\lambda)\left(\lambda-a_{k}\right)\right)}=x+v_{k} t+\Phi_{k}, \quad k=1, \ldots, n
$$

where

$$
\Phi_{k}=\varphi_{k}+\varphi_{k}^{o}
$$

\section{Soliton Geometric Phases}

6.1. Different Approaches to Soliton Geometric Phases. Now we investigate a link between the angle-map obtained above, the Abel-Jacobi map, and geometric phases. There are three approaches: complex splitting of the spectrum, averaging and method of complex phase function $\varphi$, and the $\tau$-function approach. The latter will be discussed in Sect. 7.

Definition 6.1. We define soliton geometric phases as follows:

$$
\Delta \varphi=\oint_{C} d_{a}(\varphi) .
$$

Here $d_{a}$ is the differential and $C$ is a closed curve in the space of parameters $(a)$.

The integral in (6.1) depends on the choice of a connection in the space of parameters. For example, in case of a flat connection, we get the following result. 
Theorem 6.2. Some of the soliton geometric phases coincide with the phase shift of the $k^{\text {th }}$ soliton of the multi-soliton solution and can be described by the singular integral

$$
\Delta \varphi_{k}=\frac{1}{2} \oint_{L_{k}} \frac{d \lambda}{\left(M(\lambda)\left(\lambda-a_{k}\right)\right)}, \quad k=1, \ldots, n .
$$

Here $L_{k}$ is a cycle over the cut $\left[0, a_{k}\right]$ on the Riemann surface.

Proof. In what follows we consider time $(t)$ as a parameter of the problem and our curve in $t$-space is a curve from $(-\infty)$ to $(\infty)$.

Lemma 6.3. Asymptotic reduction results, in particular, in the splitting of every element of the discrete spectrum $a_{k}$ into a pair of pure imaginary points $\left(-i \alpha_{k}, i \alpha_{k}\right)$. The phase function $\varphi_{k}$ is defined on the covering space of the generalized Jacobian.

In what follows we demonstrate the general approach using the (d)NLS and $\mathrm{KdV}$ equations as examples.

We use the change of variables

$$
\xi_{j}^{2}(x)=-\lambda_{j}(x), \quad \alpha_{k}^{2}=-a_{k}, \quad \rho_{k}=2 \alpha_{k}
$$

in the $\mathrm{KdV}$ case, and

$$
\xi_{j}^{2}(x)=\frac{\left(\lambda_{j}-b_{1}\right)}{\left(b_{2}-\lambda_{j}\right)}, \quad \alpha_{k}^{2}=\frac{\left(a_{k}-b_{1}\right)}{\left(b_{2}-a_{k}\right)}, \quad \rho_{k}=\sqrt{\left(b_{2}-a_{k}\right)\left(a_{k}-b_{1}\right)}
$$

in the NLS case, to formally integrate the basic expression occurring in the right-hand side of (5.10):

$$
\int \frac{d \lambda_{j}}{2 M\left(\lambda_{j}\right)\left(\lambda_{j}-a_{k}\right)}=\frac{1}{2 \rho_{k}} \log \left|\frac{\left(\xi_{j}-\alpha_{k}\right)}{\left(\xi_{j}+\alpha_{k}\right)}\right| .
$$

Lastly we obtain (6.2) as a sum of values of the (5.13) for $d_{k} \rightarrow \infty$ and $d_{k} \rightarrow-\infty$. Note that we take the integral in (5.13) with different signs since for $d_{k} \rightarrow \infty$ and $d_{k} \rightarrow-\infty$ it is defined on different sheets of the Riemann surface

$$
P=\frac{\log \left(\lambda-a_{k}\right)}{2 M(\lambda)}
$$

For the splitting approach, we want to define limit points $\lambda_{j}^{1}$ (or $\xi_{j}^{1}$ ) of the angle-map so that (6.5) and (5.14) are real-valued for every $j=1, \ldots, n$.

There are two choices, namely

$$
\begin{aligned}
\xi_{j+1}^{1} & =-\alpha_{j} \text { for } j=0, \ldots, k-1 \text { where } \alpha_{0}=0 \\
\xi_{j}^{1} & =\alpha_{j} \text { for } j=k+1, \ldots, n,
\end{aligned}
$$

and

$$
\begin{aligned}
\xi_{j+1}^{1} & =\alpha_{j} \text { for } j=0, \ldots, k-1, \text { where } \alpha_{0}=0, \\
\xi_{j}^{1} & =-\alpha_{j} \text { for } j=k+1, \ldots, n,
\end{aligned}
$$

which we take for $\left(d_{k} \rightarrow-\infty\right)$ and $\left(d_{k} \rightarrow \infty\right)$. This gives two different relations

$$
\frac{1}{2 \rho_{k}}\left[\log \prod_{j=k+1}^{n} \frac{\left(\alpha_{j}-\alpha_{k}\right)}{\left(\alpha_{j}+\alpha_{k}\right)}-\log \prod_{j=0}^{k-1} \frac{\left(\alpha_{k}-\alpha_{j}\right)}{\left(\alpha_{j}+\alpha_{k}\right)}\right]=\varphi_{k}^{+}
$$


and

$$
\frac{1}{-2 \rho_{k}}\left[\log \prod_{j=k+1}^{n} \frac{\left(\alpha_{j}-\alpha_{k}\right)}{\left(\alpha_{j}+\alpha_{k}\right)}-\log \prod_{j=0}^{k-1} \frac{\left(\alpha_{k}-\alpha_{j}\right)}{\left(\alpha_{j}+\alpha_{k}\right)}\right]=\varphi_{k}^{-}
$$

between the phases of the solitons $\left(\varphi_{k}^{+}, \varphi_{k}^{-}\right)$and the scattering data (parameters of the system), namely the discrete spectrum $a_{j}$. Thus for the integral (6.2), we get the following expression

$$
\Delta \varphi_{k}=\varphi_{k}^{+}-\varphi_{k}^{-}
$$

which coincides with the asymptotic formula given in [2] for the soliton phase shift if one sets

$$
\alpha_{j}=\chi_{n-j}, \quad j=1, \ldots, n .
$$

Remark 6.4. If some of the points from (6.7) and (6.8) coincide with each other, then we obtain an interaction between only some of the solitons of the $n$-soliton solution (partial phase shifts).

Realizing the soliton phase shift in the general context of geometric phases takes the mystery out of this phenomena and gives an example of a phase in the case of infinite period.

The second approach is based on consideration of the complex phase function $\varphi$. We return to the basic expression for the soliton angle representation (5.13) and consider the spectrum $a_{k}$ as being complex and dependent on $t$. It is evident that the averaged value of $\overline{\theta_{k}}$ (for $t=0$ ) equals the phase of the soliton

$$
\left\langle\left.\bar{\theta}_{k}\right|_{t=0}\right\rangle=\lim _{X \rightarrow \infty} \frac{1}{2 X} \int_{-X}^{X} x_{k} d x=\varphi_{k}, \quad k=1, \ldots, n .
$$

Using the dependence of the system (5.13) on the parameters of the problem (namely the discrete spectrum $a_{k}$ ), we can obtain the shifts $\Delta \bar{\theta}_{k}$ (geometric phases) along closed curves in the neighborhoods of singular points in the space of parameters. (See [41] for another instance of the same phenomenon.)

Namely, switching $\alpha_{j}$ and $-\alpha_{j}$, the ends of the cycles $l_{j}$ (over the basic cuts $\left[\alpha_{j}, \alpha_{j-1}\right]$ on the Riemannian surface) by means of motion along a half circle in the complex plane centered at zero, we go over from the first type of the points (6.7) to the second (6.8). This corresponds to a loop $C=\sum C_{j}$ in the space of parameters since $\left(i \alpha_{j}\right)^{2}=\left(-i \alpha_{j}\right)^{2}=a_{j}$. Finally, integrating the equation

$$
\Delta \overline{\theta_{k}}=\sum_{j=1}^{n} \oint_{C} d_{a_{j}}\left\langle\overline{\theta_{k}}\right\rangle=\sum_{j=1}^{n} \oint_{C_{j}} d_{a_{j}} \varphi_{k}=\Delta \varphi_{k}
$$

we get a formula for the soliton geometric phases related to the invariants of the Jacobi varieties of the $n$-soliton angle representation. In this way, we get a new class of classical geometric phases for systems defined on noncompact Jacobi varieties.

In conclusion, we note that phases in the case of so-called "solitons with a finite-gap background" (when only some of the permitted zones on the Riemann surface collapse) and umbilic solitons were obtained [8] using a combination of action-angle variables for quasi-periodic solutions and for the soliton problem. 
6.2. Complex Geometric Phases. So far all of our examples have dealt with the real case. Here we apply the general method of the previous section to complex systems and use methods of complex analysis to yield information about geometric phases.

The main idea is to investigate the phase function (which is a part of the angle representation of the nonlinear problem) in the complex domain. In this way the geometric phase can be considered as a certain invariant of the Riemann surface (a level set in the phase space). On the other hand, it is a natural extension of the results obtained in the real case using averaging techniques. This approach is demonstrated using the angle representations for the $n$-soliton solutions of the focusing NLS equation and for the breather and kink-kink solutions of the sine-Gordon equation. The importance of the complex angle-representation is demonstrated already in the case of the (f)NLS equation where it describes new effect of a residual interaction between 1-soliton solutions after the asymptotic splitting of the $n$-soliton solution.

6.3. Geometric Phases for the Nonlinear Schrödinger $(f)$ Equation. To obtain $n$-soliton solutions of the (f)NLS equation, we consider the following limiting process: $\sqrt{C(\lambda)} \rightarrow \bar{C}(\lambda)$

$$
m_{4 k}, m_{4 k-1} \rightarrow a_{k}, \quad m_{4 k-2}, m_{4 k-3} \rightarrow \bar{a}_{k}, \quad k=1, \ldots, N,
$$

applied to the basic polynomial present in the first integrals

$$
P_{j}^{2}=-C\left(\lambda_{j}\right)=-\prod_{r=1}^{4 N}\left(\lambda_{j}-m_{r}\right), \quad j=1, \ldots, g
$$

of the quasi-periodic (NLS) Hamiltonian system. Here $N=(g+1) / 2, g$ is odd and $g$ is the genus of the limiting Riemannian surface.

We obtain from the quasi-periodic case, the following differential system:

$$
\begin{aligned}
& \frac{\partial \lambda_{j}}{\partial x}=2 i \frac{\prod_{k=1}^{N}\left(\lambda_{j}-a_{k}\right)\left(\lambda_{j}-\bar{a}_{k}\right)}{\prod_{i \neq j}\left(\lambda_{j}-\lambda_{i}\right)}, j=1, \ldots, g ; \quad g=2 N-1, \\
& \frac{\partial \lambda_{j}}{\partial t}=2 i D_{j} \frac{\prod_{k=1}^{N}\left(\lambda_{j}-a_{k}\right)\left(\lambda_{j}-\bar{a}_{k}\right)}{\prod_{i \neq j}\left(\lambda_{j}-\lambda_{i}\right)},
\end{aligned}
$$

describing an $n$-soliton solution of the (f) NLS equation. Here

$$
D_{j}=\left(-\sum_{l \neq j}^{g} \lambda_{l}+\sum_{k=1}^{N}\left(a_{k}+\bar{a}_{k}\right)\right) .
$$

Theorem 6.5. The systems (6.16) and (6.17) are soliton Hamiltonian systems with Hamiltonians

$$
H_{s}^{s}=\frac{\sum_{j=1}^{g}\left(e^{2 i P_{j}}-\bar{C}\left(\lambda_{j}\right)\right)}{\prod_{r \neq j}\left(\lambda_{j}-\lambda_{r}\right)}
$$

and

$$
H_{s}^{d}=\frac{\sum_{j=1}^{g} D_{j}\left(e^{2 i P_{J}}-\bar{C}\left(\lambda_{j}\right)\right)}{\prod_{r \neq j}\left(\lambda_{j}-\lambda_{r}\right)}
$$


and with first integrals

$$
P_{j}=\frac{1}{2 i} \sum_{k=1}^{N} \log \left(\lambda_{j}-a_{k}\right)\left(\lambda_{j}-\bar{a}_{k}\right) \quad j=1, \ldots, g,
$$

defined on the cotangent bundle $T^{*}\left((\Re \times \ldots \times \mathfrak{R}) / \sigma_{g}\right)$, where the Riemann surface has the form

$$
\mathfrak{R}: P=\frac{1}{2 i} \sum_{k=1}^{N} \log \left(\lambda-a_{k}\right)\left(\lambda-\bar{a}_{k}\right) .
$$

Theorem 6.6. The Hamiltonian system (6.19) has a system of action-angle variables. As $(t \rightarrow \infty)$ or $(t \rightarrow-\infty)$, the system of angle variables of the $n$-soliton solution splits into $n$ 1-soliton angle variables.

Proof. From the system (6.17), we obtain the following system:

$$
\begin{aligned}
\frac{1}{2 i} \frac{\dot{\lambda}_{j}}{\left(\lambda_{j}-a_{r}\right)\left(\lambda_{j}-\bar{a}_{r}\right)} & =D_{j} \frac{\prod_{k \neq r}^{N}\left(\lambda_{j}-a_{k}\right)\left(\lambda_{j}-\bar{a}_{k}\right)}{\prod_{i \neq j}\left(\lambda_{j}-\lambda_{i}\right)}, \quad r=1, \ldots, N, \\
\frac{1}{2 i} \frac{\dot{\lambda}_{j}}{\left(\lambda_{j}-a_{r}\right)\left(\lambda_{j}-\bar{a}_{r+1}\right)} & =D_{j} \frac{\prod_{k \neq r}^{N}\left(\lambda_{j}-a_{k}\right) \prod_{k \neq r+1}^{N}\left(\lambda_{j}-\bar{a}_{k}\right)}{\prod_{i \neq j}\left(\lambda_{j}-\lambda_{i}\right)}, \quad r=1, \ldots, N-1 .
\end{aligned}
$$

Summing up the above equations with respect to $j$ from 1 to $(2 N-1)$, we obtain

$$
\begin{aligned}
\theta_{r} & =-\frac{1}{4 \operatorname{Im}\left(a_{r}\right)} \sum_{j=1}^{g} \int_{\lambda_{j}^{o}}^{\lambda_{j}}\left(\frac{1}{\left(\lambda_{j}-a_{r}\right)}-\frac{1}{\left(\lambda_{j}-\bar{a}_{r}\right)}\right) d \lambda_{j}=x+v_{r} t \\
r & =1, \ldots, N,
\end{aligned}
$$

and

$$
\begin{aligned}
\theta_{r+N} & =\frac{1}{2 i\left(a_{r}-\bar{a}_{r+1}\right)} \sum_{j=1}^{g} \int_{\lambda_{j}^{o}}^{\lambda_{j}}\left(\frac{1}{\left(\lambda_{j}-a_{r}\right)}-\frac{1}{\left(\lambda_{j}-\bar{a}_{r+1}\right)}\right) d \lambda_{j} \\
& =x+v_{r} t, \quad r=1, \ldots, N-1 .
\end{aligned}
$$

Here

$$
\begin{aligned}
v_{r} & =2 \operatorname{Re}\left(a_{r}\right), \quad r=1, \ldots, N ; \\
v_{r+N} & =a_{r}+\bar{a}_{r+1}, \quad r=1, \ldots, N-1 .
\end{aligned}
$$

Now take the real parts of the expressions (6.24) and (6.25),

$$
\begin{gathered}
\operatorname{Re}\left(\theta_{r}\right)=d_{k}+\left(v_{r}-v_{k}\right) t, \quad r \neq k, r=1, \ldots, N, \\
\operatorname{Re}\left(\theta_{r+N}\right)=d_{k}+\frac{\left(\left(v_{r}-v_{k}\right)+\left(v_{r+1}-v_{k}\right)\right)}{2} t, \quad r \neq k, r=1, \ldots, N-1,
\end{gathered}
$$

and apply asymptotic reduction along the direction $d_{k}=X+v_{k} t(1 \leqq k \leqq N)$ in a way similar to Theorem 5.4. We use the following expression:

$$
\frac{1}{4 \operatorname{Im}\left(a_{r}\right)} \sum_{j=1}^{g} \log \left|\frac{\lambda_{j}-\bar{a}_{r}}{\lambda_{j}-a_{r}}\right| \text {. }
$$


This yields the splitting of (6.24) into $N$ 1-soliton angle representations with their corresponding phase functions. Therefore, the angle representation (6.24) describes $N$-soliton solutions.

Remark 6.7. The Eqs. (6.25) describe the interaction between 1-solitons that remain after the asymptotic splitting of the $n$-soliton solution. It makes the case of (f)NLS equation different from other soliton equations and might have important applications in nonlinear optics.

To deal with the limit argument part of (6.24), we return to the formulae connecting $U$ to the solutions $Q$ from Sect. 2,

$$
U=-2 \sum_{j=1}^{g} \lambda_{j}+\sum_{k=1}^{N}\left(a_{k}+\bar{a}_{k}\right), \quad U=i \frac{\partial}{\partial x} \log Q .
$$

Integrating this expression and considering limit (6.29), we obtain an arbitrary constant in the phase of the exponent. We put this constant equal to the argument part of the phase function corresponding to (6.29).

In what follows, we describe the action variables conjugate to the variables (6.25). We introduce the action-function

$$
S=\sum_{j=1}^{g} \int_{\lambda_{j}^{o}}^{\lambda_{j}} P_{j} d \lambda_{j}=\frac{1}{2 i} \sum_{j=1}^{g} \int_{\lambda_{j}^{o}}^{\lambda_{j}} \sum_{k=1}^{N} \log \left(\lambda_{j}-a_{k}\right)\left(\lambda_{j}-\bar{a}_{k}\right) d \lambda_{j},
$$

and define conjugate variables $I_{k}$ and $\theta_{k}$ as follows:

$$
\begin{gathered}
I_{k}=-\left(\operatorname{Im}\left(a_{k}\right)\right)^{2}, \quad k=1, \ldots, N, \\
\theta_{k}=-\frac{\partial S}{\partial I_{k}} .
\end{gathered}
$$

6.4. Geometric Phases for the sine-Gordon Equation. In case of the sine-Gordon equation, the associated quasi-periodic system $[22,9]$ has the form

$$
\begin{aligned}
& \frac{\partial \lambda_{j}}{\partial x}=2\left(1+G_{N-1}\left(\lambda_{j}\right)\right) \frac{\sqrt{-\lambda_{j} C\left(\lambda_{j}\right)}}{\prod_{i \neq j}\left(\lambda_{j}-\lambda_{i}\right)}, \\
& \frac{\partial \lambda_{j}}{\partial t}=2\left(1-G_{N-1}\left(\lambda_{j}\right)\right) \frac{\sqrt{-\lambda_{j} C\left(\lambda_{j}\right)}}{\prod_{i \neq j}\left(\lambda_{j}-\lambda_{i}\right)},
\end{aligned}
$$

defined on the symmetric product of the $n$ copies of the hyperelliptic curve

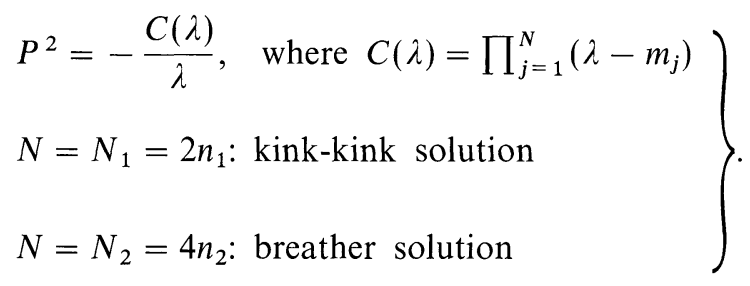

Here

$$
G_{N-1}(\lambda)=\sum_{l=0}^{N-1} g_{l} \lambda^{N-1-l}
$$


with the coefficients from

$$
G(\lambda)=\prod_{j=1}^{N}\left(\lambda-\lambda_{j}\right)=\sum_{r=0}^{N} g_{r} \lambda^{N-r} .
$$

Using different limiting processes $\sqrt{C(\lambda)} \rightarrow \bar{C}(\lambda)$ :

$$
\begin{array}{r}
m_{2 k}, m_{2 k-1} \rightarrow a_{k}, \quad a_{k}=\left(i\left(\alpha_{k}\right)\right)^{2}, \quad k=1, \ldots, n_{1}, \\
\left(n_{1}\right) \text { kink-kink solution, } \\
m_{4 k}, m_{4 k-1} \rightarrow a_{k}, \quad m_{4 k-2}, m_{4 k-3} \rightarrow \bar{a}_{k}, \quad k=1, \ldots, n_{2},
\end{array}
$$$$
\left(n_{2}\right) \text { breather solution, }
$$

we can obtain different soliton solutions.

Theorem 6.8. The system (6.35) is a Hamiltonian system with the Hamiltonian

$$
\begin{aligned}
& H_{s}^{s}=\frac{\sum_{j=1}^{n}\left(e^{2 \sqrt{-\lambda_{j}} P_{J}}-\bar{C}\left(\lambda_{j}\right)\right)\left(1+G_{N-1}\left(\lambda_{j}\right)\right)}{\prod_{r \neq j}\left(\lambda_{j}-\lambda_{r}\right)}, \\
& H_{s}^{d}=\frac{\sum_{j=1}^{n}\left(e^{2 \sqrt{-\lambda_{j}} P_{j}}-\bar{C}\left(\lambda_{j}\right)\right)\left(1+G_{N-1}\left(\lambda_{j}\right)\right)}{\prod_{r \neq j}\left(\lambda_{j}-\lambda_{r}\right)},
\end{aligned}
$$

and the complete system of first integrals

$$
P_{j}=\frac{\log \bar{C}\left(\lambda_{j}\right)}{2 \sqrt{-\lambda_{j}}}, \quad j=1, \ldots, N
$$

and which has a system of action-angle variables. Here $n=n_{1}$ or $n=n_{2}$.

Proof. Corresponding action-angle variables can be described as follows in the kink-kink case:

$$
I_{k}=a_{k}, \quad \theta_{k}=-\frac{\partial S}{\partial I_{k}}=\frac{1}{2} \sum_{j=1}^{n_{1}} \int_{\lambda_{j}^{o}}^{\lambda_{j}} \frac{1}{\left(\lambda_{j}-a_{k}\right)} \frac{d \lambda_{j}}{\sqrt{-\lambda_{j}}}, \quad k=1, \ldots, n_{1},
$$

in the breather case:

$$
\begin{gathered}
I_{k}=a_{k}, \quad \theta_{k}=\frac{1}{2} \sum_{j=1}^{n_{2}} \int_{\lambda_{j}^{o}}^{\lambda_{j}} \frac{1}{\left(\lambda_{j}-a_{k}\right)} \frac{d \lambda_{j}}{\sqrt{-\lambda_{j}}}=w_{k} x+v_{k} t, \quad k=1, \ldots, n_{2}, \\
I_{k+n_{2}}=\bar{a}_{k}, \quad \theta_{k+n_{2}}=\frac{1}{2} \sum_{j=1}^{n_{2}} \int_{\lambda_{j}^{o}}^{\lambda_{j}} \frac{1}{\left(\lambda_{j}-\bar{a}_{k}\right)} \frac{d \lambda_{j}}{\sqrt{-\lambda_{j}}}=\bar{w}_{k} x+\bar{v}_{k} t, \quad k=1, \ldots, n_{2} .
\end{gathered}
$$

Here

$$
w_{k}=\left(1-\frac{1}{4 a_{k}}\right), \quad v_{k}=\left(1+\frac{1}{4 a_{k}}\right) .
$$

Corollary 6.9. The soliton (kink-kink) angle representation for the sine Gordon equation is equivalent to a particular case of the $\mathrm{KdV}$ and $(d)$ NLS representation $\left(n_{1}=2 n_{2}\right)$. 
Corollary 6.10. The soliton (breather) angle representation for the sine-Gordon equation is transformed into the kink-kink representation if one takes $\left(n_{1}=2 n_{2}\right)$ and $\left(a_{2 k}, a_{2 k+1}\right) \rightarrow b_{k}, k=1, \ldots, n_{2}$, and then $b_{k} \rightarrow\left(a_{k}, \bar{a}_{k}\right)$.

A remaining question is: what separates these cases? In the 2-dimensional case, this question was addressed in $[16,25]$ where special real action-angle variables were used to describe the breather-kink-antikink transition through a homoclinic orbit.

Below we introduce angle-representations for such orbits in the $n$-dimensional case.

Theorem 6.11. Soliton (kink-kink) and breather angle representations for the sine Gordon equation are separated by the following singular class of "resonant solutions":

$$
\begin{aligned}
\theta_{k} & =\frac{1}{2} \sum_{j=1}^{n_{1}} \int_{\lambda_{j}^{o}}^{\lambda_{j}} \frac{1}{\left(\lambda_{j}-b_{k}\right)} \frac{d \lambda_{j}}{\sqrt{-\lambda_{j}}}, \quad k=1, \ldots, n_{2}, \\
\theta_{k+n_{2}} & =\frac{1}{2} \sum_{j=1}^{n_{1}} \int_{\lambda_{j}^{o}}^{\lambda_{j}} \frac{1}{\left(\lambda_{j}-b_{k}\right)^{2}} \frac{d \lambda_{j}}{\sqrt{-\lambda_{j}}}, \quad k=1, \ldots, n_{2},
\end{aligned}
$$

obtained from (6.45). The phase function can be obtained using the fact that

$$
\theta_{k+n_{2}}=\frac{\partial \theta_{k}}{\partial b_{k}}
$$

Here $b_{k}, k=1, \ldots, n_{2}$ are negative real numbers and $N_{1}=2 n_{1}=4 n_{2}$.

We finally combine (6.44) and (6.45) to obtain the angle representation for the $\left(N_{1}+N_{2}\right)$-soliton solution of the sine Gordon equation. phases.

Investigating this representation, one obtains the corresponding geometric

\section{Soliton Geometric Phases and the $\tau$-Function}

The importance of the angle variables for the investigation of soliton geometric phases can be demonstrated using the connection between the Riemann theta function $\Theta_{N}$ and the $\tau$-function, which play a basic role in the description of quasi-periodic and soliton solutions of the $\mathrm{KdV}$ equation [38]. (Here $N$ is the genus of the tori, which is a level set in the phase space of the quasi-periodic problem.)

These solutions can be described as follows:

$$
\begin{aligned}
U_{N} & =-2 \frac{\partial^{2}}{\partial x^{2}} \log \Theta_{N} \text { and } U_{S}=-2 \frac{\partial^{2}}{\partial x^{2}} \log \tau \\
\Theta_{N} & =\sum_{M_{1}, \ldots, M_{N}=-\infty}^{\infty} \exp \left(\frac{1}{2} \sum_{k, l=1}^{N} A_{k l} M_{k} M_{l}+\sum_{l=1}^{N} M_{l} v_{l}\right), \\
\tau & =\sum_{M_{1}, \ldots, M_{N}=0,1} \exp \left(\sum_{k<l}^{N} \Gamma_{k l} M_{k} M_{l}+\sum_{l=1}^{N} M_{l} \beta_{l}\right) .
\end{aligned}
$$


Here, $A_{k l}$ are elements of the symmetric matrix of real periods of holomorphic di rentials,

$$
\Gamma_{k l}=\left(\frac{\left(\alpha_{k}-\alpha_{l}\right)}{\left(\alpha_{k}+\alpha_{l}\right)}\right)^{2},
$$

and $v_{l}, \beta_{l}$ are angle variables in the quasi-periodic and soliton cases respectively. It can be shown [38] that $\Theta_{N}$ is transformed into the $\tau$-function as a result of the limiting process (4.1). Moreover, the principal part of $\Theta_{N}$ (singular theta-function) which generates $\tau$-function is defined by the following choice of indices $\left(M_{1}, \ldots, M_{N}=0,1\right)$, and it can be described using angle variables of the quasiperiodic problem [6]. At the same time a correspondence is established between matrices $A$ and $\Gamma$, which enables one to define geometric phases in the quasiperiodic case by mapping the signiture of the phase shift of the soliton

$$
\Delta \varphi_{k}=\sum_{j=k+1}^{n} \Gamma_{k j}-\sum_{j=0}^{k-1} \Gamma_{j k}
$$

back into the singular theta-function

$$
\begin{aligned}
\Delta \theta_{k} & =\sum_{j=k+1}^{n} A_{k j}-\sum_{j=0}^{k-1} A_{j k}-A_{k k}=\sum_{j=k+1}^{n} A_{j k}-\sum_{j=0}^{k-1} A_{j k}-A_{k k} \\
& =\sum_{r=0}^{N-1} C_{r k} \sum_{j=1}^{n} \oint_{l_{j}} \frac{\lambda_{j}^{N-1-r}}{\sqrt{R\left(\lambda_{j}\right)}} d \lambda_{j}=\sum_{r=0}^{N-1} C_{r k} \Delta v_{r} \quad k=0, \ldots, N-1 .
\end{aligned}
$$

An interesting fact is that $\Delta \theta_{k}$ phases constructed in this way coincide with those connected with the braid group [6].

These last phases can be described briefly as follows. Introduction of the angle-representation in the quasi-periodic case enables one to consider integrable systems as systems associated with a torus bundle with the base $S_{N}$ (where $N=2 n+1$ ), moduli space of complex polynomials

$$
Q(\lambda)=-F(\lambda) M(\lambda)=-\prod_{j=1}^{2 n+1}\left(\lambda-y_{j}\right)
$$

having no multiple roots, and a fiber $J$ (the $n$-dimensional complex Jacobian) defined by the Abel-Jacobi map. In [6] it is shown that systems of this type provide an example of a new class of (nonlocal) geometric phases. The idea is to show that transporting the system along certain closed curves in the base of the torus bundle (the space of parameters), could lead to a change of the homology basis, resulting in nontrivial shifts of the averaged angle variables of the problem. Such a change is realised under the action of the element from the symplectic representation of the braid group $B_{N}$

$$
A_{*}:\left(B_{N}=\pi_{1}\left(S_{N}\right)\right) \rightarrow S p(n, Z) .
$$

The phase shifts considered above are related to the generators of this representation.

The $\tau$-function is a solution of the Hirota equation and has an important interpretation in terms of Kac-Moody algebras. Therefore, using the approach described above, geometric phases can be introduced in the general case of integrable systems. 


\section{Soliton Geometric Asymptotics}

Another application of the machinery developed here is to geometric asymptotics. In particular, the quantum and classical phases are linked.

In what follows, we study the quantization of Hamiltonians that are quadratic in $P$ since the quantization procedure in the exponential case (as with our soliton Hamiltonians above), is not clear. However, the work here suggests that this can nevertheless be done, at least on the level of geometric asymptotics and phases. We demonstrate our approach using the $\mathrm{KdV}$ equation.

To obtain soliton geometric asymptotics, we first consider the quantum problem corresponding to the finite gap (quasi-periodic) Hamiltonians [9] for the $\mathrm{KdV}$ equation,

$$
H^{s}=-\sum_{j=1}^{n} \frac{\left(P_{j}^{2}+C_{2 n+1}\left(\lambda_{j}\right)\right)}{\prod_{r \neq j}\left(\lambda_{j}-\lambda_{r}\right)}
$$

and

$$
H^{d}=-\sum_{j=1}^{n} \frac{2\left(-\sum_{l \neq j} \lambda_{l}-\sum_{k=1}^{2 n+1} m_{k}\right)\left(P_{j}^{2}+C_{2 n+1}\left(\lambda_{j}\right)\right)}{\prod_{r \neq j}\left(\lambda_{j}-\lambda_{r}\right)} .
$$

These are quadratic Hamiltonians

$$
H=\frac{1}{2} \sum_{j=1}^{n} g^{j j} P_{j}^{2}+V\left(\lambda_{1}, \ldots, \lambda_{n}\right)
$$

defined on the $T^{*}\left((\mathfrak{R} \times \ldots \times \mathrm{R}) / \sigma_{n}\right)$. (Here $\mathfrak{R}$ is the Riemann surface (6.36).) In accordance with $[5,6,7]$ we use the functions $g^{j j}$ given by

$$
g^{j j}=-\frac{1}{\prod_{r \neq j}\left(\lambda_{j}-\lambda_{r}\right)}
$$

and

$$
g^{j j}=\frac{\left(-\sum_{l \neq j} \lambda_{l}-\sum_{k=1}^{2 n+1} m_{k}\right)}{\prod_{r \neq j}\left(\lambda_{j}-\lambda_{r}\right)}
$$

as components of the Riemannian metric and construct an operator of Laplace-Beltrami type and then the stationary Schrödinger equation

$$
\nabla^{j} \nabla_{j} U+w^{2}(E-V) U=0
$$

defined on the $n$-dimensional Jacobi variety. (Here $\nabla^{j}$ and $\nabla_{j}$ are covariant and contravariant derivatives defined by the tensor $g^{j j}$ and $w$ and $E$ are parameters.)

Theorem 8.1. The quantum equation (8.6) corresponding to the Hamiltonian (8.1) (or (8.2)) can be reduced to a system of $n$ ODE's connected with each other by means of $n$ constants.

Proof. We substitute a solution of the form $U=\prod_{j=1}^{n} U_{j}\left(\lambda_{j}\right)$ into Eq. (8.6) and divide the result obtained by $U w^{2}$. This results in the following formula:

$$
\sum_{j=1}^{n} \frac{R_{j}\left(\lambda_{j}\right) G_{j}}{\prod_{r \neq j}\left(\lambda_{j}-\lambda_{r}\right)}=-E
$$


Here $G_{j}=1$ and $G_{j}=\left(-\sum_{l \neq j} \lambda_{l}-\sum_{k=1}^{2 n+1} m_{k}\right)$ in cases (8.4) and (8.5) respectively. At the same time the following system of equations is satisfied:

$$
-\frac{1}{w^{2} U_{j}}\left(\frac{\partial^{2} U_{j}}{\partial \lambda_{j}^{2}}+w^{2} C_{2 n+1}\left(\lambda_{j}\right) U_{j}\right)=R_{j}\left(\lambda_{j}\right) \quad j=1, \ldots, n .
$$

The expression (8.7) is an interpolation formula and thus shows that the functions $R_{j}(\lambda)$ are the same polynomial

$$
R_{j}(\lambda)=R(\lambda)=w_{1} \lambda^{n-1}+w_{2} \lambda^{n-2}+\ldots+w_{n}, \quad w_{1}=-E
$$

with constant coefficients.

Thus, the system (8.8) is the desired system of ODE's connected with each other by means of separation constants $\left(w_{k}\right)$ which are coefficients of the polynomial $R(\lambda)$.

Now we establish a link between Eq. (8.6) and the initial nonlinear problem by means of geometric asymptotics. Let

$$
\begin{aligned}
U & =\sum_{r} A_{r}\left(\lambda_{1}, \ldots, \lambda_{n}\right) \exp \left[i w S_{r}\left(\lambda_{1}, \ldots, \lambda_{n}\right)\right] \\
& =\sum_{r} \prod_{j=1}^{n} U_{r j}\left(\lambda_{j}\right)=\sum_{r} \prod_{j=1}^{n}\left(A_{j}\left(\lambda_{j}\right) \exp \left[i w S_{r j}\left(\lambda_{j}\right)\right]\right),
\end{aligned}
$$

which is a function of several complex variables defined on the covering space of the Riemann surface of the problem. Substituting (8.10) in (8.8), equating coefficients for $w$ and $w^{2}$ and integrating, we obtain the system of equations

$$
\begin{gathered}
A_{j}^{2}=\frac{c}{\frac{\partial S^{o}}{\partial \lambda_{j}}}=\frac{c}{\sqrt{C_{2 n+1}\left(\lambda_{j}\right)}}, \quad c=\text { const }, \\
S^{o}=\sum_{j=1}^{n} S_{j}^{o}\left(\lambda_{j}\right)=\sum_{j=1}^{n} \int_{\lambda_{j}^{o}}^{\lambda_{j}} \sqrt{C_{2 n+1}\left(\lambda_{j}\right)} d \lambda_{j}, \quad j=1, \ldots, n,
\end{gathered}
$$

which result in the following form of geometric asymptotics [5]:

$$
U=\sum_{r} \frac{A_{o}}{\prod_{j=1}^{n}\left(C_{2 n+1}\left(\lambda_{j}\right)\right)^{\frac{1}{4}}} \exp \left[i w \sum_{j=1}^{n} \int_{\lambda_{j}^{o}}^{\lambda_{j}} \sqrt{C_{2 n+1}\left(\lambda_{j}\right)} d \lambda_{j}+i \Delta S_{r j}\right] .
$$

Let us consider the special class of quasi-periodic solutions of the initial $\mathrm{KdV}$ equation defined by the following choice of basic polynomial:

$$
C_{2 n+1}(\lambda)=(-\lambda) \prod_{k=1}^{n}\left(\lambda-a_{k}\right)^{2}+R(\lambda)
$$

which depends on exactly $2 n$ parameters $\left(a_{r}, w_{k}\right)$.

Definition 8.2. We call solutions from this class presoliton solutions.

Theorem 8.3. $n$-soliton solutions correspond to the case when all coefficients $w_{k}$ of the polynomial $R(\lambda)$ from (8.14) are equal to zero. 
Proof. When $R(\lambda)=0$, the action function has the form

$$
S=\sum_{j=1}^{n} \int_{\lambda_{j}^{o}}^{\lambda_{j}}\left(\sqrt{-\lambda} \prod_{k=1}^{n}\left(\lambda_{j}-a_{k}\right)\right) d \lambda_{j}
$$

corresponding to the standard soliton Hamiltonian defined on the $T^{*}\left((\mathfrak{R} \times \ldots \times \mathfrak{R}) / \sigma_{n}\right)$, where $\mathfrak{R}$ is a degenerate Riemann surface (pinched torus)

$$
P^{2}=(-\lambda) \prod_{k=1}^{n}\left(\lambda-a_{k}\right)^{2} .
$$

On the other hand if we set $R(\lambda)=0$ in the quantum system (8.8), then every equation can be considered independently. It is clear that as $\lambda_{j}$ approaches $a_{j}$ (or $\left.a_{j+1}\right)$ the $j^{\text {th }}$ equation is dominated by the term $\left(\lambda_{j}-a_{j}\right)$ (or $\left.\left(\lambda_{j}-a_{j+1}\right)\right)$, meaning that the system (8.8) splits into $n$ copies of 1 -dimensional soliton quantum equations. Thus the initial equation $(8.8)$ (with $R(\lambda)=0$ ) corresponds to the class of $n$-soliton solutions of the $\mathrm{KdV}$ equation.

Corollary 8.4. If $R(\lambda)=0$, then formulae (8.13) describes soliton geometric asymptotics with

$$
U=\sum_{j} \frac{A_{o}}{\prod_{j=1}^{n}\left(M\left(\lambda_{j}\right) \prod_{r=1}^{n}\left(\lambda_{j}-a_{r}\right)\right)^{\frac{1}{2}}} \exp \left[i w \sum_{j=1}^{n} \int_{\lambda_{j}^{o}}^{\lambda_{j}} M\left(\lambda_{j}\right) \prod_{r=1}^{n}\left(\lambda_{j}-a_{r}\right) d \lambda_{j}\right] .
$$

Definition 8.5. In what follows, we consider the coefficients $w_{k}$ of the $R(\lambda)$ to be action-variables of the problem. In this case angle-variables (conjugate to $w_{k}$ ) have the form

$$
\vartheta_{k}=-\frac{\partial S}{\partial w_{k}}=-\sum_{j=1}^{n} \int_{\lambda_{j}^{o}}^{\lambda_{j}} \frac{\lambda_{j}^{n-1-k}}{\sqrt{C_{2 n+1}\left(\lambda_{j}\right)}} d \lambda_{j}, \quad k=0, \ldots,(n-1) .
$$

We call $w_{k}$ and $\vartheta_{k}$ presoliton action-angle variables.

Note that $w_{k}$ are exactly quantum numbers of the system (8.8). Condition $R(\lambda)=0$ results in the particular form of variables $\vartheta_{k}$ denoted $\theta_{k}$,

$$
\theta_{k}=-\sum_{j=1}^{n} \int_{\lambda_{j}^{o}}^{\lambda_{j}} \frac{\lambda_{j}^{n-1-k}}{M\left(\lambda_{j}\right) \prod_{r=1}^{n}\left(\lambda_{j}-a_{r}\right)} d \lambda_{j}, \quad k=0, \ldots,(n-1)
$$

which are directly connected with the soliton angle variables (5.2) as follows:

$$
\theta_{k}=\sum_{r=1}^{n} b_{k r} \bar{\theta}_{r}, \quad k=0, \ldots,(n-1),
$$

where $b_{k r}$ are constants.

Corollary 8.6. Since the matrix $B=\left(b_{k r}\right)$ is uniquely defined with respect to the parameters $a_{r}$ a link between geometric phases (8.18) in the case of standard soliton Hamiltonians and phases in the case of Hamiltonians (4.6), (4.7) (described in Sects. 5, 6) can be expressed as follows:

$$
\Delta \theta=B \Delta \bar{\theta} .
$$

Investigating the dependence of the soliton geometric asymptotics (8.17) on the slowly changing parameters $a_{l}$, one can also obtain geometric phases in the 
quantum case. In particular, using presoliton geometric asymptotics (8.14) and presoliton phase $P^{o}=w S^{o}$ one can define soliton phase shifts in the quantum case as follows:

$$
\left(\Delta_{G} P^{\mathrm{sol}}\right)_{k}=\Delta\left(\left.\frac{\partial P^{o}}{\partial w_{k}}\right|_{R=0}\right), \quad k=1, \ldots, n,
$$

which yields the following link with geometric phases in the classical case:

$$
\Delta \theta_{k}=-\frac{1}{w}\left(\Delta_{G} P^{\mathrm{sol}}\right)_{k}, \quad k=1, \ldots, n .
$$

We note that the methods of this paper were recently applied [8] to the investigation of umbilic solitons and homoclinic orbits of the nonlinear equations. The umbilic solitons provide a geometric model for homoclinic orbits that approach low dimensional tori instead of homoclinic points as $t \rightarrow \infty$. It is also shown that the Hamiltonian flow associated with homoclinic orbits introduced by Devaney [19] for the C. Neumann problem, coincides with the soliton $x$-flow of the $\mathrm{KdV}$ equation. This leads to the introduction of homoclinic Hamiltonians and geometric phases.

Acknowledgements. We would like to thank the referee for excellent suggestions. We want to express our deep gratitude to D. McLaughlin for very helpful discussions.

\section{References}

1. Ablowitz, M.J., Segur, H.: Asymptotic solutions of the Korteweg-de Vries equation. Stud. Appl. Math. 57, 13-44 (1977)

2. Ablowitz, M.J., Segur, H.: Solitons and the Inverse Scattering Transform. Philadelphia: SIAM 1981

3. A'Campo, N.: Tresses, monodromie et le group symplectique. Comment. Math. Helvetici 54, 318-327 (1979)

4. Adler, M., Van Moerbeke, P.: Completely integrable systems, Kac-Moody Lie algebras and curves. Adv. Math. 38(3), 267-317 (1980)

5. Alber, M.S.: On integrable systems and semiclassical solutions of the stationary Schrödinger equations. Inverse Problems 5, 131-148 (1989)

6. Alber, M.S.: Complex geometric asymptotics, geometric phases and nonlinear integrable systems. Studies in Math. Phys. vol. 3, pp. 415-427. Amsterdam: North-Holland, Elsevier 1992

7. Alber, M.S.: Hyperbolic geometric asymptotics. Asymptotic Analysis 5, 161-172 (1991)

8. Alber, M.S., Marsden, J.E.: Geometric phases and monodromy at singularities. (to appear)

9. Alber, M.S., Alber, S.J.: Hamiltonian formalism for finite-zone solutions of integrable equations. C.R. Acad. Sci. Paris 301, 777-781 (1985)

10. Arnold, V.I.: Dynamical Systems. Berlin, Heidelberg, New York: Springer 1988

11. Arnold, V.I.: A remark on the branching of hyperelliptic integrals as functions of the parameters. Func. Anal. Appl. 2, 187-189 (1968)

12. Berry, M.V.: Quantal phase factors accompanying adiabatic changes. Proc. R. Soc. Lond. A 392, 45 (1984)

13. Berry, M.V.: Classical adiabatic angles and quantal adiabatic phase. J. Phys. A: Math. Gen. 18, 15 (1985)

14. Berry, M.V., Hannay, J.H.: Classical non-adiabatic angles. J. Phys. A: Math. Gen. 21, L325 (1988)

15. Berry, M.V.: Quantum adiabatic anholonomy. Lectures given at the Ferrara School of Theoretical Physics on "Anomalies, defects, phases ... ", June 1989, (to be published by Bibliopolis (Naples)) 
16. Bishop, A.R., McLaughlin, D.W., Solerno, M.: Global coordinates for the breather-kink (antikink) sine-Gordon phase space: An explicit separatrix as a possible source of chaos. Phys. Rev. A 40, 11, 6463-6469 (1989)

17. Calogero, F., Degasperis, A.: Spectral transform and solitons. Amsterdam: North-Holland 1982

18. Deift, P., Li, L.C., Tomei, C.: Matrix factorizations and integrable systems. Comm. Pure Appl. Math. 443-521 (1989)

19. Devaney, R.: Transversal homoclinic orbits in an integrable system. Am. J. Math. 100, 631-642 (1978)

20. Duistermaat, H.J.: On global Action-Angle Coordinates. Comm. Pure Appl. Math. 23, 687 (1980)

21. Ercolani, N., McKean, H.P.: Geometry of $\mathrm{KdV}(4)$ : Abel sums, Jacobi variety, and theta function in the scattering case. Invent. Math. 99, 483-544 (1990)

22. Ercolani, N., Forest, M., McLaughlin, D.W., Montgomery, R.: Hamiltonian Structure for the Modulation Equations of a sine-Gordon Wavetrain. Duke Math. J. 55, (4) 949-983 (1987)

23. Ercolani, N., Forest, M., McLaughlin, D.W.: Notes on Melnikov integrals for models of the periodic driven pendulum chain. Preprint (1989)

24. Ercolani, N., Forest, M.: The Geometry of Real sine-Gordon Wavetrains. Commun. Math. Phys. 99, 1-49 (1985)

25. Ercolani, N., McLaughlin, D.W.: Toward a Topological Classification of Integrable PDE's. In: Ratiu, T. (ed.), Proc. of a Workshop on The Geometry of Hamiltonian System, MSRI Publications vol 22, Berlin, Heidelberg, New York: Springer, 1991, pp. 111-130

26. Flaschka, H., McLaughlin, D.W.: Canonically Conjugate Variables for the Korteweg-de Vries Equation and the Toda Lattice with Periodic Boundary Conditions. Prog. Theor. Phys. 55(2), 438-456 (1976)

27. Flaschka, H., McLaughlin, D.W., Forest, M.G.: Multiphase Averaging and the Inverse Spectral Solution of the Korteweg-de Vries Equation. Comm. Pure Appl. Math. 33, 739 (1980)

28. Flaschka, H., Newell, A.C., Ratiu, T.: Kac-Moody Lie Algebras and Soliton Equations II, III. Physica 9D, 300-332 (1983)

29. Forest, M.G., McLaughlin, D.W.: Spectral theory for the periodic sine-Gordon equation: A concrete viewpoint. J. Math. Phys. 23(7), 1248-1277 (1982)

30. Forest, M.G., McLaughlin, D.W.: Modulation of Sinh-Gordon and Sine-Gordon Wavetrains. Studies Appl. Math. 68, 11-59 (1983)

31. Francoise, J.-P.: Arnold's Formula for Algebraically Completely Integrable Systems. Bull. AMS (New Series) 17, 2, 301-303 (1987)

32. Guillemin, V., Sternberg, S.: Geometric Asymptotics. Math. Surveys 14, Providence, Rhode Island: AMS 1977

33. Guillemin, V., Sternberg, S.: The Gelfand-Cetlin system and the quantization of the complex Flag Manifolds. J. Funct. Anal. 52(1), 106-128 (1983)

34. Hasegawa, A., Kodama, Y.: Guiding-center soliton in optical fibers. Optical Letters 15, 24, 1443-1445 (1990)

35. Knörrer, H.: Singular fibres of the momentum mapping for integrable Hamiltonian systems. J. Reine. Ang. Math. 355, 67-107 (1984)

36. Lax, P.D., Levermore, C.D.: The small Dispersion Limit of the Korteweg-de Vries Equation, I, II, III. Comm. Pure Appl. Math. 36(2), 253-290, 571-593, 809-830 (1983)

37. Marsden, J.E., Montgomery, R., Ratiu, T.: Cartan-Hannay-Berry Phases and Symmetry. Contemp. Math. 97, 279 (1989); see also Mem. AMS Vol. 436 (1990)

38. McKean, H.P.: Integrable Systems and Algebraic Curves. Lecture Notes in Mathematics Berlin, Heidelberg, New York: Springer 1979

39. McKean, H.P.: Theta functions, solitons, and singular curves. In: Byrnes, C.I. (ed.), PDE and Geometry, Proc. of Park City Conference 237-254 (1977)

40. McKean, H.P., Trubowitz, E.: Hill's operator and hyperelliptic function theory in the presence of infinitely many branch points. Comm. Pure Appl. Math. 29, 143-226 (1976)

41. Montgomery, R.: The Connection whose Holonomy is the classical Adiabatic Angles of Hannay and Berry and Its Generalization to the Non-Integrable case. Commun. Math. Phys. 120, 269-294 (1988)

42. Moser, J.: Integrable Hamiltonian Systems and Spectral Theory, Academia Nazionale dei Lincei, Fermi Lecture, Pisa, 1981 
43. Mumford, D.: Tata Lectures on Theta I and II, Progress in Math. vol. 28 and vol. 43, Boston: Birkhauser 1983

44. Previato, E.: Hyperelliptic quasi-periodic and soliton solutions of the nonlinear Schrödinger equation. Duke Math. J. 52, 2 (1985)

45. Ruijsenaars, S.N.M.: Action-Angle Maps and Scattering Theory for some Finite-Dimensional Integrable Systems I. The Pure Soliton Case. Commun. Math. Phys. 115, 127-165 (1988)

46. Tracy, E.R.: Topics in nonlinear wave theory with applications. Ph.D. Thesis, University of Maryland, College Park 1984

47. Venakides, S.: The Generation of Modulated Wavetrains in the Solution of the Korteweg-de Vries Equation. Comm. Pure and Appl. Math. 38, 883-909 (1985)

48. Venakides, S., Zhang, T.: Periodic Limit of Inverse Scattering. Preprint, Duke University, 1991

49. Weinstein, A.: Connections of Berry and Hannay type for moving Lagrangian submanifolds. Adv. Math. 82, 2, 133-159 (1990)

50. Witten, E.: Quantum Field Theory and the Jones Polynomials. Commun. Math. Phys. 121, 351-399 (1989)

Communicated by A. Jaffe 